Herd Behavior, the " Penguin Effect", and the Suppression of Informational Diffusion:

An Analysis of Informational Externalities and

Payoff Interdependency

By Jay Pil Choi

Discussion Paper No. 691 


\title{
Herd Behavior, the "Penguin Effect", and the Suppression of Informational Diffusion: \\ An Analysis of Informational Externalities and Payoff Interdependency
}

\author{
By \\ Jay Pil Choi*
}

November 1993

revised. March 1994

*Department of Economics. Columbia University, U.S.A. and

CentER for Economic Research. Tilburg University, The Netherlands

The author is currently visiting the CentER for Economic Research at Tilburg University. whose hospitality and financial support are gratefully acknowledged. 


\begin{abstract}
This paper analyzes a technology adoption process in which the effect of informational spillover interacts with network externalities. It is shown that the interplay of informational externalities and payoff interdependency induces risk averse and clustering behavior in the technology adoption process. Our analysis differs from the herd behavior literature in focusing on how the herd behavior of subsequent users influences the initial adoption decision. The mechanism through which herd behavior is generated is also quite different. Herd behavior in this paper stems from each agent's desire to inhibit the revelation of new information which can be used in a way detrimental to her. rather than from each agent's effort to free-ride on information contained in the decisions made by predecessors. Finally, the model suggests a new perspective on standard-setting committees. Their role is to limit deliberately the effect of information flows, rather than to serve as a forum for exchange of information and negotiation.
\end{abstract}




\section{Introduction}

There are many economic activities that produce, in addition to their directly intended benefits, payoff-relevant information as a byproduct. The price charged by a monopolist facing an unknown demand curve creates, besides immediate current profits, valuable information about the demand, which can be used for future pricing decisions (Aghion et al. [1991]). Consumers who purchase a drug of unknown quality receive information about its true quality as well as its health benefits (Grossman, Kihlstrom and Mirman [1977]). Learning-by-doing production yields information which enhances the efficiency of future production (Arrow [1962]).

Often, as in the case of knowledge spillover in $R \& D$, those who were not directly engaged in the information-generating activities can have access to the same information as the producers themselves, thus enabling agents to learn from the experiences of others. If the payoff for an individual decision-maker depends only on her own actions, this informational externality will be typically ignored in the formulation of an individual's decision-making, which causes an incongruence of the individually and socially optimal decision rules. However, when the payoff of an agent is jointly determined by everyone else's action, the effect of informational spillover needs to be recognized even at the level of individual decision-making. The reason is that every agent has to predict the informational consequences of her actions on those of the subsequent decision-makers which, in turn, affect her own payoff. This paper addresses such a problem in the context of irreversible technology choice with network externalities. ${ }^{1}$

\footnotetext{
${ }^{1} \operatorname{Rob}(1991)$ is another paper dealing with informational externality in a multi-agent framework. He analyzes a dynamic entry process into a new industry under demand uncertainty. Since he considers a competitive market structure with free entry in a model of continuum of agents, there is no strategic interaction among agents. Informational externalities and payoff interdependence operate only at the aggregate level. The main result of his paper is that due to the informational externalities. the equilibrium rate of entry at any given point in time is lower than the socially optimal one. Caplin and Leahy (forthcoming) is another example of a competitive model with informational externalities.
} 
We present a simple model of technology adoption that allows us to analyze the interplay of informational externalities and payoff interdependency. There are two new technologies available for adoption. Each consumer chooses one of the available technologies. Initially, uncertainty prevails about the true values of the technologies. The nature of the uncertainty is such that the values of the technologies can be ascertained only by actually using them (Rosenberg [1982] and Zeira [1986]). There is a fixed number of potential users of the competing technologies with identical preferences. We allow the possibility that each may learn from the others' experiences. For simplicity, it is assumed that once a technology is used by someone, the value of it becomes public knowledge, thereby creating informational externalities. Imagine, for instance, that firms contemplate adopting a new technology, the cost-reducing effectiveness of which is not completely known. The uncertainty can be resolved only by harnessing the technology. If other firms can observe profits of the implementing firm and the technology can be applied by any firm with more or less equal effectiveness, then the adoption of the technology can reveal information about its unknown characteristics to everyone. ${ }^{2}$ We also assune that technologies exhibit network externalities. This creates positive payoff interdependency: the more people adopt the same technology, the more valuable it is. ${ }^{3}$ Therefore, each adopter has to be concerned with the effect of information generated by her decision on the adoption decisions of other potential users. We start with a sequential model of technology adoption with an exogenously given order of decision-making. Then, we discuss the case of endogenous timing.

In a model of sequential technology choice, we demonstrate that once a technology is adopted and its true value is revealed, it has a significant advantage over another

\footnotetext{
${ }^{2} \mathrm{Kislev}$ and Shchori-Bachrach (1973) document such a process in the adoption of new technologies.

${ }^{3}$ See Farrell and Saloner (1986) and Katz and Shapiro (1986), for instance, for possible sources of network externalities.
} 
technology whose value is uncertain in the subsequent technology adoption process. The consequence of handicapping the adoption of the other technology is the prevalence of herd behavior in the technology adoption process. At first sight, herd behavior seems to be a natural phenomenon to expect in a model with positive feedback effect. Network externalities are undoubtedly responsible for the clustering behavior. The underlying channel through which the externalities operate, however, differs from the one in previous models of complete information (Farrell and Saloner [1985, 1986] and Katz and Shapiro [1986]). In a game of complete information about technologies, each agent is better off moving earlier rather than later due to the commitment power when the choice is irreversible. ${ }^{4}$ Moreover, the technology chosen early enjoys a strategic advantage in the subsequent adoption due to the installed-base effect which sets the herd behavior in motion. Therefore, what distinguishes the competing technologies in terms of strategic position is the number of subscribers who are already in the network.

Herd behavior in our model, in contrast, is driven by the fear of being stranded. Experimentation with a new technology is avoided because of its informational consequences accompanying the adoption of an untested technology. Consequently, each decision-maker is concerned with the number of the future subscribers who can act upon new information that can be created by her decision.

More importantly, the herd behavior of subsequent users influences the adoption decision of the agent who moves first. It will be shown that, even for risk-neutral users, there is a bias in the first choice towards a safer technology. Such individually optimal risk averse behavior, however, can induce the first adopter to make a suboptimal choice viewed

${ }^{4}$ When we endogenize the timing of adoption in Section IV, we demonstrate that each agent may prefer to wait for another user to choose first in the presence of uncertainty about the true values of the technologies. Caplin and Leahy (1993a) independently discovered the difference between crowd externalities and informational externalities. They note that "[i]n the crowd story the decisions ... are complementary... In the information story the decisions are strategic substitutes." 
in terms of collective welfare. The initial suboptimal choice can pose a very serious problem especially when the choice is perpetuated by the subsequent users' herd behavior. If this possibility is foreseen, a new role for standard-setting committees can be called for. Before the adoption process starts, the concerned parties may opt for an early standardization as a commitment device. Setting standards can effectively deprive the future potential adopters of the opportunity to respond to new information by constraining the feasible choice set. Thereby, correct incentives can be restored by relieving the initial adopters of the risk of being "orphaned." This is in sharp contrast to the prevailing view of committees which portrays them as a forum for exchanging technical information among potential adopters.

The paper relates to two strands of literature: herd behavior and network externalities. First of all, the overall technology adoption behavior in our paper resembles herd behavior as analyzed by Banerjee (1992) and Scharfstein and Stein (1990) among others. They are interested in the efficiency of markets in aggregating decentralized information. Banerjee, for instance, develops a model of sequential choice in which the history of the past decisions made by the predecessors, but not the basis of those decisions. is observable to each new decision-maker. In his model, herd behavior can arise naturally as an outcome of rational decision-making because each agent suspects that her predecessors had private information and tries to free-ride on it.

Despite similarities in the overall behavior, there are crucial differences between this paper and Banerjee's (1992). First of all, the underlying mechanism through which herd behavior is generated is quite different. In his model, herd behavior stems from each agent's effort to free-ride on information contained in the decisions already made. In our model, prevention of the adoption of an untested technology is instead driven by each agent's desire to inhibit the revelation of new information to the followers who can use the newly revealed information in a way which is detrimental to the creator of the information. Whereas informational externalities operate only in the forward direction in Banerjee's 
model, the interplay of payoff interdependence and informational spillover induces externalities in both the forward and backward direction, thereby creating interesting dynamics. As a consequence, in his model, the first investor's decision is an optimal one and the possible inefficiency arises only from the behavior of the followers. In contrast, the focus of our model is on how the herd behavior of subsequent users can affect the first mover's technology choice. Second, in Banerjee's model, information is passively received. His main question is whether the market efficiently aggregates existing information that is dispersed across economic agents. In our model, information is created as a result of adopting a new technology. ${ }^{5}$ Therefore, we ask whether there is correct incentive to create information. Finally, his model is devoid of any strategic elements since each agent's payoff is completely independent of the choices made by each subsequent agent in the decision process. Therefore, in order to elicit herd behavior. he assumes that the returns from each decision are realized only after everybody has made their choice. If the reward associated with each option were revealed immediately after its adoption as in our model, there would be no herd behavior.

Scharfstein and Stein (1990) prescribe a similar behavior in an agency model of investment. They consider a situation in which managers $A$ and $B$ invest sequentially based on private signals which are correlated across agents. The main result is that manager B who makes an investment decision after observing the choice of manager A. pays too much attention to what manager $\mathrm{A}$ has done, leading to inefficient herd behavior. They attribute this type of behavior to the distortion in incentives, which they call the "sharing the blame" effect. The compensation scheme based on relative performance induces one's payoff to depend on the other agent's action and introduces payoff interdependency between agents. In this regard, Scharfstein and Stein's (1990) is closer

5 Zeira (1987) is another paper in which the investment process is accompanied by resolution of "structural uncertainty." 
to our paper. Their primary focus, however, is with the effect of reputational concerns on the follower's investment decision whereas our paper analyzes the systematic inefficiency in the initial choice. ${ }^{6}$

The paper is also closely related to the network externalities literature. Farrell and Saloner (1985) consider a model of standard-setting in which there is uncertainty about the preferences of the other potential adopters. The equilibrium adoption behavior is characterized as "bandwagon." They prove that there is a bias against the adoption of a new technology, which they call "excess inertia." This paper provides another channel in which a bias against a new technology can be created, namely, uncertainty regarding the inherent value of the new technology.

The remainder of the paper is organized in the following way. Section II sets up a basic model of sequential technology adoption. The equilibrium behavior is analyzed in Section III. The implications of endogenizing the adoption timing are discussed in Section IV, in which a new persnective on the role of standard-setting committees is presented. The concluding section includes possible extensions and limitations of the model.

\section{I1. The Sequential Choice Model}

There are $\mathrm{N}$ risk-neutral users who contemplate adopting one of the two incompatible technologies A and B. We assume that there is a predetermined order according to which consumers choose one of the two technologies. The option of delay is not allowed. The consequences of relaxing these assumptions will be discussed later. We index consumers according to their order of choice. Each consumer has the same valuation for each technology which exhibits network externalities. We assume that the values of adopting each technology depend only on the intrinsic value of the technology

6It should be mentioned that Scharfstein and Stein are also aware of the possibility that reputational concerns could induce even manager A to behave differently. See footnote 10 in their paper. 
and the final size of its network. We envision a situation in which all users have the opportunity to adopt the new technology at essentially the same time. Therefore, following Farrell and Saloner (1985), we ignore interim payoffs. More specifically, the net benefits for an individual choosing technology A (B) is given by $\alpha(\beta)+v_{n}$ when there are $\mathrm{n}$ people choosing the same technology including the individual herself. We interpret $v_{n}$ as the positive network benefit which is increasing in $n$. By normalizing $v_{1}=$ 0 , we can interpret $\alpha(\beta)$ as the stand-alone or intrinsic benefit of technology A (B). Each technology has the characteristic of "experience" good; the true stand-alone values of the two technologies are uncertain before they are actually used. They are, however, known to be drawn from distribution functions $\mathrm{F}$ and $\mathrm{G}$, respectively. For simplicity, we assume that they are independently distributed and have continuous and everywhere positive density on support $[0, \infty)$. We further assume that once a technology is used by anyone, its true value is revealed perfectly and can be observed by other potential users. As a result, each agent benefits from observational experience, i.e. is able to observe other agents' decisions and learn from their consequences; experience is technology-specific but not user-specific. The adoption of technology is irreversible. Since the individual decisions are made only once, the model is devoid of any motives for experimentation at the level of individual decision-making, which allows us to focus on the issue of informational externalities. Our aim in this paper is to investigate how the informational extemalities interact with network externalities to induce herd behavior and create biases against the adoption of a new technology.

\section{The Equilibrium Decision Rule}

To understand how the existence of network externalities influences the technology adoption process under uncertainty, it is useful to analyze first the benchmark case in which there are no network externalities involved. Since the value of each technology is independent of other users' decisions in the absence of network externalities, the dynamics 
of decentralized decision are trivial. User 1 will choose the technology which has the higher expected value. Suppose that technology A was adopted by user 1 and its value was revealed to be $\alpha$. Then, user 2's optimal decision depends on the comparison of $\alpha$ and $E(\beta)$. If $E(\beta)>\alpha$, user 2 adopts technology $B$. Then, the value of technology $B, \beta$, will also be known. All subsequent users will make decisions with the benefit of complete information and choose the technology whose value is revealed to be higher. If $\mathrm{E}(\beta) \leq \alpha$, user 2 chooses the same technology as user 1 and there is no information revelation on the value of technology B. Since the subsequent users face exactly the same problem as user 2 , there will be no one choosing technology $\mathrm{B}$. The equilibrium adoption behavior in this game has the following property; for the values of the technologies to be fully disclosed in the decentralized adoption game in equilibrium, the first two users have to select different technologies. As will be shown, this property carries over to the case where network externalities are present. The reason is that with network externalities, user 2 has a stronger incentive than any of her followers to adopt a different technology from the predecessor's. Intuitively, with network externalities, the more people have chosen the same technology before, the more attractive it becomes, leading to less incentive to try another technology.

Let us investigate the equilibrium decision rule in the presence of network externalities. It will be shown that network externalities introduce a significant bias against experimenting with a new technology compared with the benchmark case. At the outset, it should be stressed that the main source of this bias is not the installed base effect as in Farrell and Saloner (1986). The reason is as follows. Since the second user has the strongest incentive to try a new technology, we only have to examine her willingness to experiment. If there is a sufficient number of potential users, for user 2 the difference in installed base between technologies A and B (of only one) is almost negligible. This suggests that the underlying force behind the bias is from the forward looking behavior, i.e., the fear of being stranded by future users. Therefore, what really matters is the 
number of users who make the adoption decision after rather than before user 2 . Of course, eventually the installed base effect will dominate. However, what sets the herd behavior in motion initially is the fear of being stranded.

To make the argument more precise, we proceed by backward induction. Suppose that all users leading up to the final user $\mathrm{N}$ have adopted technology $\mathrm{A}$ and there has been no experimenting with the other technology $B$. User $\mathbf{N}$ will then experiment with the new technology $B$ if and only if

$$
E(\beta)>\alpha+v_{N}
$$

In other words, she will try a new technology if and only if its expected value is high enough to offset the installed base advantage of technology $A$, which is $\left(v_{N}-v_{1}\right)=v_{N}$ by the normalization assumption. ${ }^{7}$

With the knowledge of the decision rule for the final user $\mathrm{N}$, let us investigate the incentive for the penultimate user $(\mathrm{N}-1)$ to experiment assuming that only technology $A$ has been adopted by all previous users. If condition (1) holds, the value of choosing the same technology as the predecessors' is given by $\alpha+v_{\mathrm{N}-1}$; the last user will choose technology $\mathrm{B}$ and therefore the network externality benefit will be only $\mathrm{v}_{\mathrm{N}-1}$. If she experiments with a new technology, her expected payoff would be $E(\beta)+\left[1-\mathrm{G}\left(\alpha+\mathrm{v}_{\mathrm{N}-1}-\mathrm{v}_{2}\right)\right] \mathrm{v}_{2}$. The formula for the expected payoff can be explained as follows. When she adopts technology $B$, the value of technology $\beta$ will be revealed to the final user. User $N$ will follow suit if and only if the revealed value of technology B satisfies the following condition:

$$
\beta+v_{2}>\alpha+v_{N-1}
$$

Otherwise, she will be the lone adopter of technology B. Therefore, the probability that the last user will follow her in the adoption of technology and confer on her the network benefit of $v_{2}$ is given by $\left[1-\mathrm{G}\left(\alpha+\mathrm{v}_{\mathrm{N}-1}-\mathrm{v}_{2}\right)\right]$. By comparing the two payoffs, we

7 Without loss of generality, we assume that if potential users have no clear preference between the two technologies, they adopt technology A. 
conclude that if the final user $\mathbf{N}$ finds it optimal to experiment with a new technology $\mathrm{B}$, so will the penultimate user $(\mathrm{N}-1)$.

Suppose that condition (1) does not hold. Then, the (N-1)th user knows that if she does not try a new technology, the final user will not, either. Therefore, the value of choosing technology $A$ is given by $\alpha+v_{N}$. If she experiments with a new technology $B$, her expected payoff would be the same as before, i.e., $E(\beta)+\left[1-G\left(\alpha+v_{N-1}-v_{2}\right)\right] v_{2}$. By comparing the two payoffs, we can derive the condition for experiment for the (N-1)th user when condition (1) is violated.

Combining the two cases analyzed above, we can conclude that the penultimate user (N-1) will adopt a new technology B if and only if

$$
E(\beta)>\alpha+\left[v_{N}-v_{2}\right]+G\left(\alpha+v_{N-1}-v_{2}\right) v_{2} .
$$

Since condition (3) is less stringent than (1), user (N-1) has a bigger incentive to experiment than user $\mathbf{N}$. Intuitively, in comparison to the final user, the penultimate user faces a smaller size of installed base. Moreover, the rev technology chosen by the penultimate user can potentially have a larger network; whereas user $\mathrm{N}$ is bound to be the only subscriber of the new technology when she is the first one to experiment, the penultimate user can have another subscriber of the new technology if the final user follows her.

We can fold further back and analyze the (N-2)nd user in a similar way. She will experiment if and only if $E(\beta)>\alpha+\left[v_{N}-v_{3}\right]+G\left(\alpha+v_{N-1}-v_{3}\right) v_{3}$.

In general, the formula for the kth user to try a new technology B is given by

$$
E(\beta)>\alpha+\left[v_{N}-v_{N-k+1}\right]+G\left(\alpha+v_{N-1}-v_{N-k+1}\right) v_{N-k+1} \text {. }
$$

By the network externality assumption, $v_{N-k+1}$ is decreasing in $k$. $G($.$) is increasing in$ its argument and $0 \leq \mathrm{G}() \leq$.1 . The RHS of inequality (3), therefore, is strictly increasing in $\mathrm{k}$, which implies that the condition for experimenting with a new technology is harder to satisfy, the later in the adoption queue a user is placed. This means that there will be no 
trial of technology B unless it is tried by user 2. Consequently, the condition for technology $\mathrm{B}$ ever being adopted by any user is given by

$$
E(\beta)>\alpha+\left[v_{N}-v_{N-1}\right]+G(\alpha) v_{N-1} .
$$

We can summarize the arguments made above in the following proposition:

Proposition 1. Suppose that the first user adopted technology A, the value of which tumed out to be $\alpha$. The condition for the second user to adopt technology B is given by:

$$
E(\beta)-\alpha>\left[v_{N}-v_{N-1}\right]+G(\alpha) v_{N-1} \text {. }
$$

If technology B is used, the true value of B will be known. Therefore, all users after user 2 will adopt the ex post better technology since each technology has the same installed base of 1 and the subgame after both technologies have been adopted, is played with complete information [see Farrell and Saloner (1985) for more details]. Otherwise, technology B will never be adopted and its true value will remain unknown. ${ }^{8}$

In other words, when the value of technology $\mathrm{A}$ is known to be $\alpha$. for the other technology to be tried, its expected value should exceed the value of technology A by $\Psi(\alpha)$ $=\left[v_{N}-v_{N-1}\right]+G(\alpha) v_{N-1}$. This implies that given the distribution of $\beta, G($.$) , there is a$ critical value of technology $\mathrm{A}$, at which user 2 is indifferent between experimenting with a new technology B and sticking with the same technology A. This critical value $\alpha^{*}$ is defined as

$$
E(\beta)-\alpha^{*}=\left[v_{N}-v_{N-1}\right]+G\left(\alpha^{*}\right) v_{N-1}
$$

Since LHS of (6) is strictly decreasing in $\alpha^{*}$ and RHS is strictly increasing in $\alpha^{*}$, there is a unique $\alpha^{*}$ that satisfies $(6)$. It is immediate that $\alpha^{*}<\mathrm{E}(\beta)$ since $\left[\mathrm{v}_{\mathrm{N}}-\mathrm{v}_{\mathrm{N}-1}\right]>0$ by the network externality assumption and the fact that $\mathrm{G}($.$) is nonnegative. We can interpret the$

${ }^{8}$ By measuring the bias against the adoption rule in the absence of network externalities, we do not imply that the individual decision rule in that case is a socially optimal one. Even without network externalities, there will be an underproduction of information due to informational spillover. What we are measuring is the extent to which network externalities exacerbate the problem. 
difference $E(\beta)-\alpha^{*}=\left[v_{N}-v_{N-1}\right]+G\left(\alpha^{*}\right) v_{N-1}$ as the bias against experimenting with a new technology or the advantage bestowed on the technology already adopted and whose true value is known to potential adopters. The bias consists of two components. We interpret the first term $\left(v_{N}-v_{N-1}\right)$ as the installed base disadvantage for a new technology. The second term represents an interplay between network and informational externalities and is interpreted as the fear of being "left out." To see this, suppose that there is no uncertainty on the value of the new technology B. Then, for technology B to be adopted by user 2 , its value needs to exceed $\alpha$ only by $\left(v_{N}-v_{N-1}\right)$, which represents the difference in the ultimate network sizes between choosing A and B when the first user is already committed to technology A. The second term appears when there is uncertainty about the true value of the untested technology. Adoption of a new technology reveals information about it which will be incorporated in the subsequent users' adoption decision. When the value of the technology tums out to be lower than $\alpha$, the user of the new technology will be stranded by the other users. This risk of being stranded as a result of information revelation serves as an obstacle for the adoption of a new technology. We expect the magnitude of the first term to be relatively small, especially when $N$ is very large and the network benefit function is concave in the size of network. We can therefore conclude that the fear of being left out, rather than the disadvantage of the installed base, is largely responsible for the bias against adopting a new technology with uncertain value.

With slight modifications of the model, the experimentation bias in Proposition 1 can be reinterpreted as excess inertia in the transition from one technology to another. Suppose that currently technology A is industry standard. As in Farrell and Saloner (1985), imagine a situation in which the firms decide sequentially whether to change to a new technology B. Then, proposition 1 suggest that for the new technology to be adopted by someone, its expected value has to exceed the value of the current standard by a significant amount. Farrell and Saloner derive the excess inertia from incomplete information regarding other users' preferences. This paper provides another mechanism 
through which excess bias against a new technology is created, namely, uncertainty about its true value in conjunction with the diffusion of information created by actually using it.

Next we analyze how an increase in the uncertainty about the untested technology, G, can influence this adoption bias. To analyze the effect of a mean preserving spread (MPS) on the magnitude of the bias, let the riskiness of the distribution function $\mathrm{G}(\beta ; \theta)$ be parametrized by $\theta$, a higher $\theta$ representing a greater riskiness of the distribution (Rothschild and Stiglitz [1970]). We impose the following regularity restriction on the distribution function.

\section{Single-Crossing-at-the-Mean Condition (SCM):}

The distribution functions of $\beta$ corresponding to two different levels of riskiness $\theta$ and $\theta^{\prime}$ with $\theta^{\prime}>\theta$ intersect only once at their mean, i.e., $F(\beta ; \theta)-F\left(\beta ; \theta^{\prime}\right) \frac{>}{<} 0$ according to $\beta_{<}^{>} E(\beta)$.

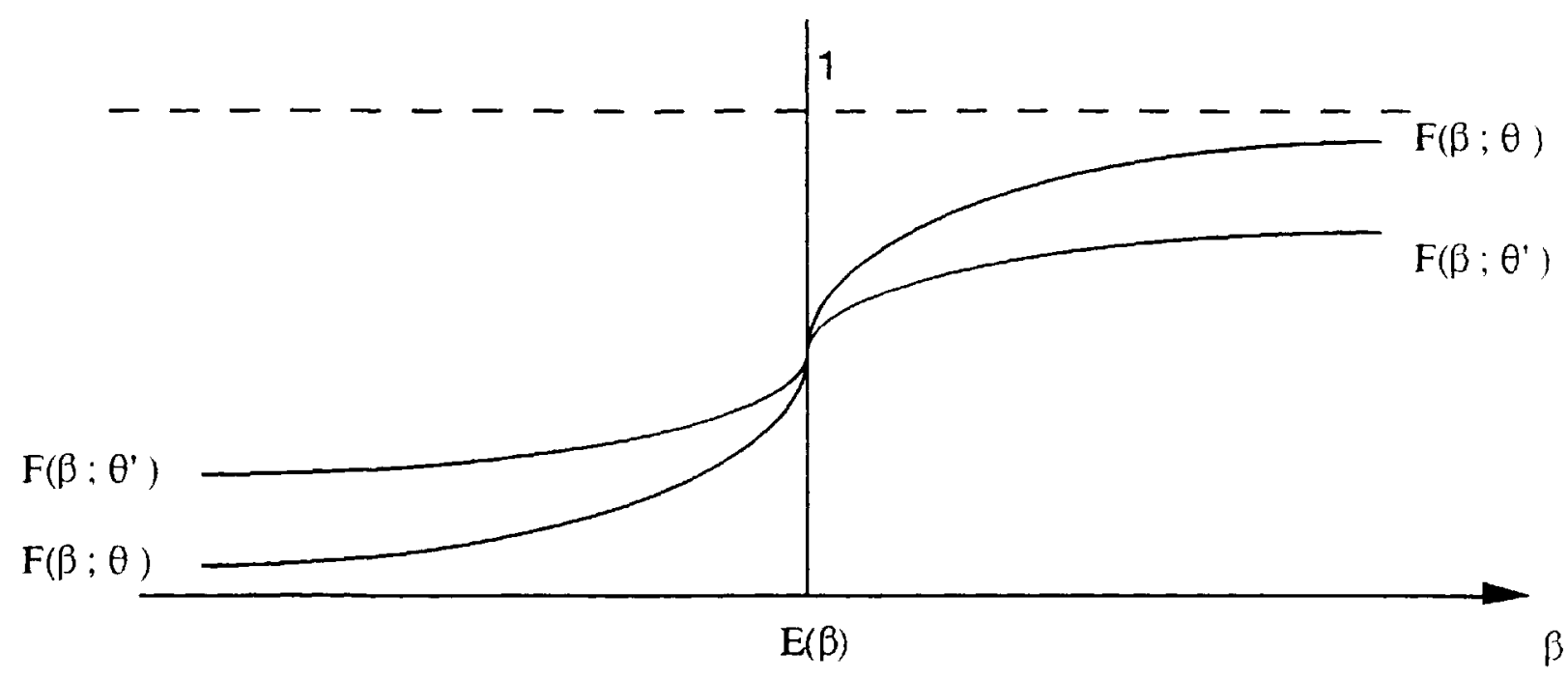

Figure 1:Mean Preserving Spread with Single-Crossing-at-the-Mean Condition

${ }^{9}$ For instance, normal distribution and uniform distribution satisfy our regularity condition if the variances are taken as riskiness parameter $\theta$. Note that our condition is weaker than the ones imposed in Bhattarcharya and Mookherjee (1986) in that we are not imposing a symmetry condition across their mean. 
Proposition 2. Let $\alpha^{*}(\theta)$ be the critical value defined in (6) corresponding to the level of riskiness $\theta$. Then, $\alpha^{*}(\theta)$ is decreasing in $\theta$. In other words, the experimentation bias, $E(\beta)-\alpha^{*}(\theta)$, is increasing with a MPS in the distribution of the new technology.

Proof. Let $\alpha^{*}(\theta)$ and $\alpha^{*}\left(\theta^{\prime}\right)$ be the critical values corresponding to the riskiness levels of $\theta$ and $\theta^{\prime}$, respectively. Then,

$$
\begin{aligned}
& E(\beta)-\alpha^{*}(\theta)=\left[v_{N}-v_{N-1}\right]+G\left(\alpha^{*}(\theta) ; \theta\right) v_{N-1} \\
& E(\beta)-\alpha^{*}\left(\theta^{\prime}\right)=\left[v_{N}-v_{N-1}\right]+G\left(\alpha^{*}\left(\theta^{\prime}\right) ; \theta^{\prime}\right) v_{N-1}
\end{aligned}
$$

Suppose the contrary, i.e. that $\alpha^{*}(\theta)<\alpha^{*}\left(\theta^{\prime}\right)$ and $\theta<\theta^{\prime}$. Then, $\mathrm{G}\left(\alpha^{*}\left(\theta^{\prime}\right) ; \theta^{\prime}\right)<$ $\mathrm{G}\left(\alpha^{*}(\theta) ; \theta\right)$ since we have $\alpha^{*}(\theta)+\mathrm{G}\left(\alpha^{*}(\theta) ; \theta\right) \mathrm{v}_{\mathrm{N}-1}=\alpha^{*}\left(\theta^{\prime}\right)+\mathrm{G}\left(\alpha^{*}\left(\theta^{\prime}\right) ; \theta^{\prime}\right) \mathrm{v}_{\mathrm{N}-1}$. However, $\mathrm{G}\left(\alpha^{*}\left(\theta^{\prime}\right) ; \theta^{\prime}\right)>\mathrm{G}\left(\alpha^{*}(\theta) ; \theta^{\prime}\right)>\mathrm{G}\left(\alpha^{*}(\theta) ; \theta\right)$. The first inequality follows from the property of the cumulative distribution function and the second one from the property of MPS. Therefore, we have a contradiction. See also Figure 2. Q.E.D.

As will be shown below, Proposition 2 has a profound implication on user 1's optimal decision problem. Ceteris paribus, user 1 prefers that there be no trial of other technology since it will create a possibility of her being stranded and losing network externalities if the new technology turns out to be better than her own technology. Therefore, the decision by user 1 will be biased against risky technologies so that subsequent users are left only with such risky technologies and hence have less incentive to experiment.

Suppose that two technologies A and B have the same mean $\mathrm{m}$, but technology B is more risky in the sense of mean preserving spread. Then, we can prove the following. 


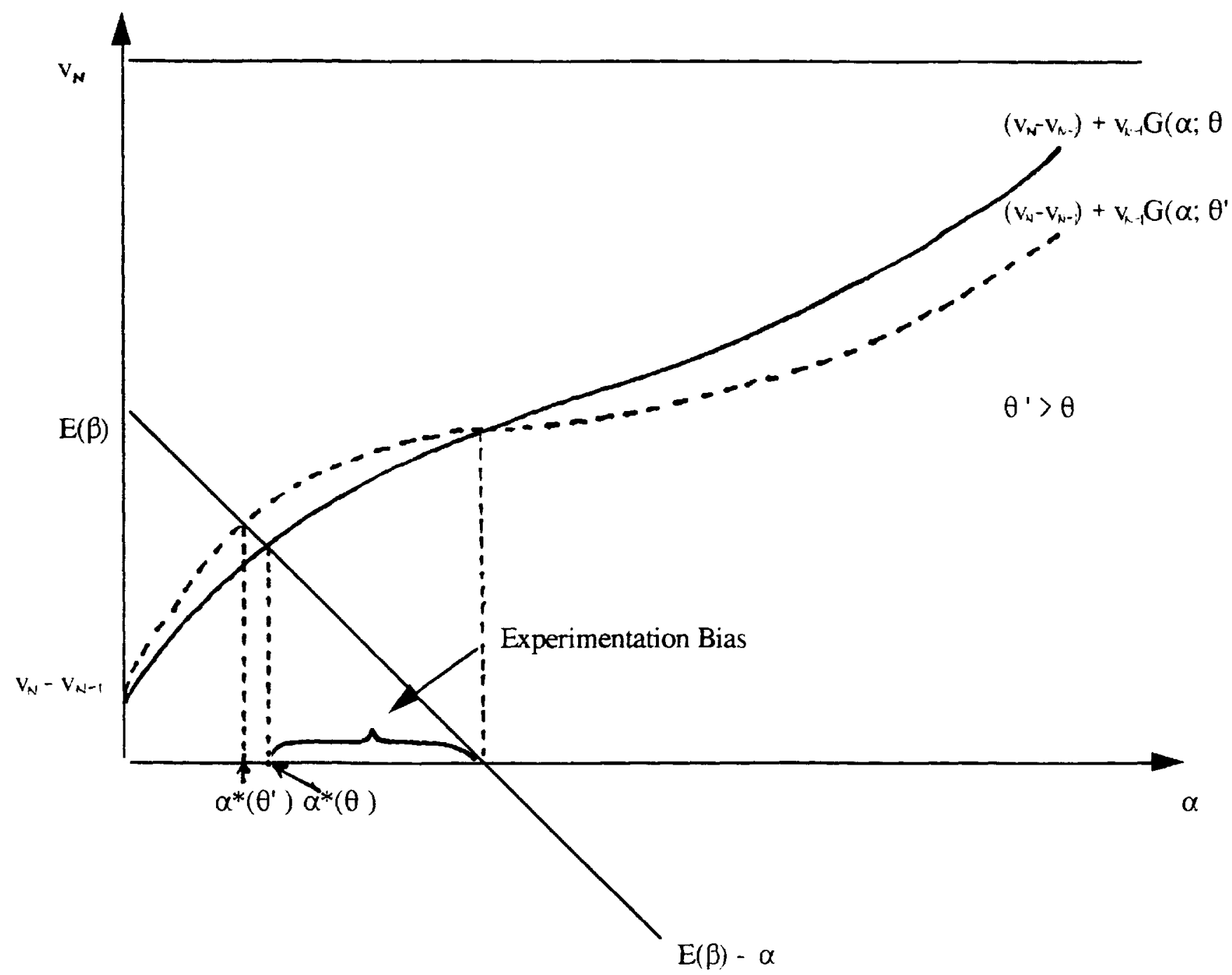

Figure 2: Mean Preserving Spread and Experimentation Bias

Proposition 3. The first user will adopt the less risky technology, A, over the more risky one, $\mathrm{B}$.

Proof. Let us define $\beta^{*}$ analogously as the critical value of technology $B$ which makes user 2 indifferent to choosing between technologies $A$ and $B$ when $B$ is adopted by user 1 .

$$
E(\alpha)-\left[v_{N}-v_{N-1}\right]=\beta^{*}+F\left(\beta^{*}\right) v_{N-1}
$$

Then, by proceeding as in the proof of Proposition 2, we can prove that $\alpha^{*}<\beta^{*}<\mathrm{m}$.

Let $\Psi_{\mathrm{A}}$ and $\Psi_{\mathrm{B}}$ be the values of choosing technologies $\mathrm{A}$ and $\mathrm{B}$, respectively, for the first adopter. Then, we have 


$$
\Psi_{\mathrm{A}}=\mathrm{E}(\alpha)+\left[1-\mathrm{F}\left(\alpha^{*}\right)\right] \mathrm{v}_{\mathrm{N}}+\int_{0}^{\alpha^{*}} \mathrm{G}(\alpha) \mathrm{v}_{\mathrm{N}-1} \mathrm{dF}(\alpha)
$$

The first term is the expected value of the stand-alone value of the technology. The network benefit from choosing a technology depends on how many people choose the same technology. The second term represents the network benefit in the case where there is no experimentation of the other technology, which, by definition of $\alpha^{*}$, applies if the value of technology A tums out to be higher than $\alpha^{*}$. Therefore, the network benefit will be $v_{N}$ in this event, the probability of which is $\left[1-F\left(\alpha^{*}\right)\right]$. The third term corresponds to the case where there is an experimentation with the other technology by user 2 , which occurs if $\alpha$ proves to be lower than $\alpha^{*}$. In this case the true values of the two technologies are fully revealed to the subsequent users. Since both technologies have the same installed base of 1 they are competing on a level playing field. Which technology will be chosen by subsequent users depends only on the stand-alone values. We have to consider two scenarios. If $\beta \leq \alpha$, which occurs with the protability of $G(\alpha)$, all subsequent users adopt technology A. Consequently, user 1 will have the network benefit of $v_{N-1}$. Otherwise, she will be the only user of technology $A$ and will have no network benefit.

Similarly, the value of choosing technology $B$ is given by

$$
\psi_{B}=E(\beta)+\left[1-G\left(\beta^{*}\right)\right] v_{N}+\int_{0}^{\beta^{*}} F(\beta) v_{N-1} d G(\beta)
$$

We have

$$
\Psi_{A}-\Psi_{B}=[E(\alpha)-E(\beta)]+v_{N}\left[G\left(\beta^{*}\right)-F\left(\alpha^{*}\right)\right]+v_{N-1} \int_{0}^{\alpha^{*}} G(\alpha) d F(\alpha)-\int_{0}^{\beta^{*}} F(\beta) d G(\beta)
$$

Since we are considering the mean preserving spread, $E(\alpha)=E(\beta)$. We also have $\left[\mathrm{G}\left(\beta^{*}\right)\right.$ $\left.-F\left(\alpha^{*}\right)\right]>0$ by Proposition 2. Therefore, we have 


$$
\begin{aligned}
& \Psi_{\mathrm{A}}-\Psi_{\mathrm{B}}>v_{\mathrm{N}-1}\left[\mathrm{G}\left(\beta^{*}\right)-\mathrm{F}\left(\alpha^{*}\right)\right]+v_{N-1}\left[\int_{0}^{\alpha^{*}} \mathrm{G}(\alpha) \mathrm{dF}(\alpha)-\int_{0}^{\beta^{*}} \mathrm{~F}(\beta) \mathrm{dG}(\beta)\right. \\
& =v_{N-1}\left[\int_{0}^{\beta^{x}}[1-F(\beta)] d G(\beta)-\int_{0}^{\alpha^{*}}[1-G(\alpha)] d F(\alpha)\right. \\
& >v_{N-1} \int_{0}^{\alpha^{*}}[1-F(\beta)] d G(\beta)-\int_{0}^{\alpha^{*}}[1-G(\alpha)] d F(\alpha) \\
& >v_{N-1} \int_{0}^{a^{x}}[1-G(x)][d G(x)-d F(x)] \\
& =v_{N-1}\left(\left[1-G\left(\alpha^{*}\right]\left[G\left(\alpha^{*}\right)-F\left(\alpha^{*}\right)\right]+\int_{0}^{\alpha^{*}}[G(x)-F(x)] d G(x)\right)>0\right. \\
& \text { (by integrating by parts) } \\
& \text { Q.E.D. }
\end{aligned}
$$

By the continuity argumeri, it can be shown that it is possible that an inferior technology in terms of expected value can be chosen by the first user if the alternative technology with a higher expected value has a sufficiently higher level of riskiness. In other words, the fear of being stranded induces risk averse behavior in the technology adoption. This has important ramifications for the welfare of society.

To illustrate the point, we consider the following example. Suppose that technology $\mathrm{A}$ is a derivative of a mature and well-established technology whose value has already been tested in other industries while technology $B$ is an emerging one whose value is hard to predict. For simplicity, we assume that technology $A$ has a certain value of $m_{A}$. Technology $B$ has an expected value of $m_{B}$ and is uniformly distributed with variance $\sigma^{2}$. In other words, $\beta$ is known to be distributed uniformly on $\left[m_{B}-\sqrt{3} \sigma, m_{B}+\sqrt{3} \sigma\right]$. Technology $B$ is assumed to have a higher expected value, i.e., $m_{B}>m_{A}$. It will be shown that, under plausible conditions, the lower-expected-value technology, A, will be 
chosen by the first user and all subsequent users; the higher-expected-value technology, B, is never adopted. ${ }^{10}$ To see this, suppose that technology $\mathrm{A}$ is chosen by the first user. Then, by Proposition 1, we can derive the condition for no experimentation by subsequent users.

$$
\mathrm{m}_{\mathrm{B}}-\mathrm{m}_{\mathrm{A}}<\frac{\sqrt{3} \sigma\left[\mathrm{v}_{\mathrm{N}}+\Delta_{\mathrm{N}}\right]}{2 \sqrt{3} \sigma+\mathrm{v}_{\mathrm{N}-1}}, \text { where } \Delta_{N}=\left[\mathrm{v}_{N}-\mathrm{v}_{\mathrm{N}-1}\right]
$$

Therefore, in this special case where the value of technology $\mathrm{A}$ is known before the adoption, if condition (7) is satisfied, the first user knows that there will be no experimentation when he chooses technology A. The value of choosing technology A is given by:

$$
\psi_{\mathrm{A}}=\mathrm{m}_{\mathrm{A}}+\mathrm{v}_{\mathrm{N}}
$$

In contrast, by proceeding as in the proof of Proposition 3, the value of choosing technology $\mathrm{B}$ can be derived to be:

$$
\psi_{B}=m_{B}+\frac{\left[m_{B}+\sqrt{3} \sigma\right]-\left[m_{A}-\Delta_{N}\right]}{2 \sqrt{3} \sigma} v_{N}
$$

Therefore, the no-risk technology with a lower expected value, $A$, will be adopted by user 1 if

$$
m_{B}-m_{A}<\frac{\left[\sqrt{3} \sigma-\Delta_{N}\right] v_{N}}{2 \sqrt{3} \sigma+v_{N}}=\varphi(\sigma, N)
$$

We can conclude that in the sequential adoption game, technology $\mathrm{A}$ will be chosen by everybody if conditions (7) and (10) are satisfied. Since condition (10) is more stringent than condition (7), it suffices to satisfy (10) for the lower expected technology A to be chosen by everybody. In our example, $\varphi(\sigma, N)$ defined in (10) can be considered a risk premium for the risky technology B to be adopted. It can be easily verified that $\varphi(\sigma, N)$ is increasing in $\sigma$. Therefore, the bias against the risky technology increases in its riskiness.

10This corresponds to an investment collapse in the model of Chamley and Gale (forthcoming). 
It also increases in $N$ unless $v_{N}$ is an extremely convex function in $N .{ }^{11}$ This means that the bias will be more conspicuous when there are more potential adopters. In the next section, it will be argued that this result suggests a new perspective on an early standardsetting in committees as an obstacle to the information flows among potential adopters rather than as a forum for the exchange of technological information.

\section{Endogenous Timing, the Penguin Effect, and the Role of the}

\section{Committee as a Suppressor of Informational Diffusion}

In the previous sections, we have assumed that the order of choice is exogenously given and the waiting option is not allowed. In this section, we speculate on what to expect when the timing of adoption is also at users' disposal and the order of choice is endogenously determined. In the endogenous timing framework, we can conceive of two kinds of inefficiencies. In addition to the bias against the risky technology identified in the previous section, we expect to observe the presence of penguin effect in the adoption game: each user will be reluctant to move first as long as there is a possibility that her choice may turn out to be so inferior as to make orphan her adoption. ${ }^{12}$ She may prefer to wait for another user to choose first in order to free-ride on informational externalities. We will discuss these two types of inefficiencies and their implications for the possible role of standardization committees in remedying them.

Consider the example analyzed in the previous section. Suppose that a safe technology, A, and a risky one, B, compete for adoption and that condition (10) is satisfied. If there is a cost associated with waiting due to discounting of payoffs, the most impatient user will be the first to adopt one of the available technologies. The choice made by her need not be the best one for the collective welfare. We know that there will be

${ }^{11} \mathrm{~A}$ sufficient condition for $\varphi(\sigma, N)$ to increase in $\mathrm{N}$ is that the network benefit function $\mathrm{v}_{\mathrm{N}}$ is weakly concave in $\mathbf{N}$.

12The term "penguin effect" was coined by Farrell and Saloner (1986). In their wordings, "Iplenguins who must enter the water to find food often delay doing so because they fear the presence of predators. Each would prefer some other penguin to test the waters first." 
biases against the adoption of risky technologies; whoever adopts a technology first will do so by choosing technology A. Moreover all subsequent adopters make the same choice, with the resulting payoff of $\Psi_{A}=m_{A}+v_{N}$. One advantage of sequential decisions is the possibility of utilizing new information generated in the course of the decision-making process. However, in this example no new information is generated due to strong herd behavior. To make matters worse, the agents herd on the wrong technology since the one with a lower expected value is adopted. With risk-neutral users, they will be collectively better off in terms of ex ante expected payoff if everyone chooses technology B since it gives the expected payoff of $\psi_{B}=m_{B}+v_{N}\left(>\psi_{A}\right)$.

With the endogenous timing, there is another inefficiency associated with the delay in the technology adoption. The learning mechanism in our model implies that each potential user has incentives to wait; the opportunity to observe the experiences of others provides valuable information for her own decision-making. These informational externalitics can transform the payoff structure of the adoption game into that of a waiting game. We illustrate how the "penguin effect" generates an inefficient delay in a discretetime infinite horizon model. For ease of exposition, we assume that there are two potential users. As in the previous example, technology $A$ has a deterministic value of $m_{A}$ while the value of technology $B$ is drawn from distribution function $G($.$) with E(\beta)=m_{B}$. We interpret $\alpha$ and $\beta$ as the present discounted values of the two technologies. Similarly. we denote by $\Delta$ the present discounted value each user attaches to the network externalities conferred when the other user adopts the same technology. The discount factor is given by $\delta$. Suppose that the following two conditions hold:

$$
\begin{aligned}
& \mathrm{m}_{\mathrm{B}}>\mathrm{m}_{\mathrm{A}}+\Delta \\
& \mathrm{m}_{\mathrm{B}}+\Delta<\delta \mathrm{G}\left(\mathrm{m}_{\mathrm{A}}-\Delta\right) \mathrm{m}_{\mathrm{A}}+\int_{\mathrm{m}_{\mathrm{A}} \cdot \Delta}^{\alpha} \beta+\Delta \mathrm{dG}(\beta)
\end{aligned}
$$


Condition (11) says that technology B is superior to technology A in expected value even if the other user is certain to adopt technology A. However, technology A can still be adopted if technology B is adopted first by the other user and its value turns out to be very low. More specifically, the condition for technology $A$ to be adopted after $\beta$ is revealed is:

$$
\beta+\Delta \leq \mathrm{m}_{\mathrm{A}}
$$

We next interpret condition (12). The RHS of inequality (12) represents the expected value of waiting when the other user adopts technology B in the current period. When $\beta$ is revealed to satisfy condition (13), which occurs with probability of $G\left(m_{A}-\Delta\right)$, technology A will be adopted. Otherwise, the same technology B will be adopted, the expected value of which is given by the second term in the square bracket. The LHS of (12) is the expected value of adopting technology B in the current period when the other user does the same. Therefore, condition (12) says that one prefers to wait one more period when the other user adopts technology $B$ in the current period; the option value of having an opportunity to learn from other's experience outweighs the loss of current benefits.

Taken together, conditions (11) and (12) ensures that the ex ante inferior technology A will be never adopted first in equilibrium, which enables us to abstract from the kind of inefficiency identified earlier. It can be adopted only after technology B is adopted and its value is revealed to satisfy condition (13). Since the adoption game is stationary and symmetric across players, we focus on the stationary symmetric equilibrium of the game. It can be easily verified that the unique symmetric equilibrium is characterized by mixed strategies in which each play adopts technology B with probability $p$ in each period if the other player has not adopted before. For $p$ to be an equilibrium probability of adoption, the players should be indifferent between adoption and waiting. When the other player adopts with probability $p$, the value of adopting technology $B$ is given by

$$
\mathrm{V}_{\mathrm{B}}(\mathrm{p})=\mathrm{p}\left[\mathrm{m}_{\mathrm{B}}+\Delta\right]+(1-\mathrm{p})\left[\mathrm{m}_{\mathrm{B}}+\delta \mathrm{G}\left(\mathrm{m}_{\mathrm{A}}-\Delta\right) \Delta\right]=\mathrm{m}_{\mathrm{B}}+\left[\mathrm{p}+(1-\mathrm{p}) \delta \mathrm{G}\left(\mathrm{m}_{\mathrm{A}}-\Delta\right)\right] \Delta(14)
$$


The value of waiting is

$$
V_{W}(p)=\delta\left\{p\left(G\left(m_{A^{-}}-\Delta\right) m_{A}+\int_{m A-\Delta}^{\infty} \beta+\Delta d G(\beta)+(1-p) V\right\}\right.
$$

where $\mathrm{V}$ is the equilibrium value of playing the adoption game.

The equilibrium probability of adoption $p^{*} \in(0,1)$ will be chosen so that $V_{B}\left(p^{*}\right)$ $=V_{W}\left(p^{*}\right)=V .{ }^{13}$ What is important for us is the fact that $V=V_{B}\left(p^{*}\right)=m_{B}+I p^{*}+(1-$ $\left.\left.\mathrm{p}^{*}\right) \delta \mathrm{G}\left(\mathrm{m}_{\mathrm{A}}-\Delta\right)\right] \Delta<\mathrm{m}_{\mathrm{B}}+\Delta$ since $\delta<1$ and $\mathrm{p}^{*}<1$. In other words, the players are better off if both of them relinquish the waiting option and adopt B immediately; delay in the adoption dissipates the expected payoffs to the extent that they are actually worse off from the opportunity to learn.

In a recent paper, Caplin and Leahy (1993a) construct a model of an urban real estate market which shares many features of our model. They consider a situation where a large number of distinct owners of unoccupied buildings in a given area are matched with potential tenants. There is common payoff-relevant uncertainty about sume aspect of the given area, say shoppers' willingness to visit the area. The uncertainty can only be resolved once a match is made. Following the first match, there is no residual uncertainty and information becomes public. They show that the delay until first occupancy is suboptimally long due to the informational externality. ${ }^{14}$ In our context of technology adoption, their result translates as too long a delay until first adoption. There is, however, a crucial difference between our model and theirs. Their model is devoid of any direct payoff interdependency among agents' actions. As a result, each individual cannot suffer

${ }^{13}$ It can be easily verified that there exists a unique $p^{*} \epsilon(0,1)$ such that $V_{B}\left(p^{*}\right)=V_{W}\left(p^{*}\right)=$ $\mathrm{V}$.

${ }^{14}$ In Caplin and Leahy (1993b), they apply the same framework to a situation of mass layoffs in which unemployed workers with similar characteristics face similar adjustment decisions. Once again, they derive the result that the natural rate of unemployment is suboptimally high due to informational externalities and the free rider problem. 
from the existence of informational spillover even though the delay is socially suboptimal. In other words, the delay cannot dissipate the expected payoff below the level that can be attained in the absence of informational externalities. ${ }^{15}$ The reason is that without any payoff interdependency one always has the option to ignore the existence of other agents, guaranteeing the payoffs attainable without informational externalities. Consequently, the expected payoffs cannot be improved by just obstructing the informational diffusion process. In the presence of network externalities, however, one cannot disregard the informational consequences of one's own action without its having an adverse effect on the payoffs. The revelation of new information can lead others to desert the technology to which one is committed. This fear (the "penguin effect") can prolong the first adoption to the extent that everyone is worse-off by having the opportunity to learn from others.

Farrell and Saloner (1986) provide another model of technology adoption in which the penguin effect can be present. As in this paper, the effect is created by the fear of being the sole user of a technology being contemplated for adoption. However, the mechanics of being "stranded" are different across models. In order to produce the "penguin" effect in a model of complete information, they introduce an artificial friction in the adoption process. More specifically, in their model the opportunity to adopt a new technology is assumed to present itself only once in a while. The assumption can be justified if users. for instance, contemplate adoption only when there is a breakdown of existing capital goods, which occurs stochastically. Consequently, a user who adopts a technology will not be followed immediately by everyone else even if they all share the same preference and agree on the most desired technology. In contrast, in this paper the penguin effect is

\footnotetext{
15 In fact, the equilibrium expected payoff with informational externalities will be exactly the same as the one without, meaning that all the possible gains from information spillover are dissipated by the delay caused by the free rider problem. To see this, note that the absence of payoff interdependency implies $\Delta=0$ in our framework. It is clear that in both cases the expected payoff is $m_{B}$.
} 
driven by the fact that an early adoption of untested technologies reveals new information which can be used to strand the first mover.

For both types of inefficiencies that we have identified, what prevents the agents from overcoming them is their inability to commit to not using newly revealed information against early adopters. As in the cases considered by Banerjee (1992), we can have situations in which destroying or limiting information can be socially beneficial.

One way to accomplish this objective is for every user to choose simultaneously, thereby limiting the opportunities to respond to new information revealed; they opt for collective ignorance. ${ }^{16}$ An early standardization agreement in committees can be considered such a scheme. ${ }^{17}$ Commitment to an early standard can make the adoption of alternatives less attractive and more costly, thereby insulating early adopters from the adverse effect of being stranded. Essentially, it induces everyone to choose the same, ex ante optimal technology, thus limiting the exploitation of informational diffusion.

Therefore, the role of standard-setting committees is to erect an obstacle to utilizing newly revealed information through an early commitment to a particular technology rather than to serve as a forum for exchange of information and negotiation.

Viewed in this way, this model provides a new perspective on the role of committees in standard setting which is in sharp contrast to that offered in Farrell and Saloner [1987, 1988] and Lifchus (1986). In these papers, early standardization is usually

${ }^{16}$ If I use the animal analogy from Farrell and Saloner (1986), the penguins may decide to plunge into the water at the same time (maybe hand in hand to prevent a deviant) when no one is willing to test the water first. Another solution to overcome the informational externality is a lump sum transfer to any player who invests in the first period. Gabel (1991), however, claims that "in practice side payments feature much more commonly in economic journals than in industry." Implicitly we consider situations in which the firms can meet and establish standards but cannot use side payments.

${ }^{17}$ In our model with identical preference and symmetric information, the consensus on the standard will be immediately reached. In contrast, Farrell and Saloner (1988) portray the standard-setting committees as coordinating mechanism in which the conflict between participants who have vested interests can be resolved. 
associated with a cost in the form of ill-informed decision-making and sacrifice of the waiting option since information typically arrives over time. The countervailing benefit is a longer horizon of compatibility benefits. ${ }^{18}$ In this model, on the other hand, preventing the utilization of new information is the purpose of early standardization. Consequently, when the technology chosen as a standard performs worse than expected and switching to a new standard is too costly, we cannot subjugate the situation to the expost criticism of too hasty adoption of the standard. In our model, that is exactly what standardization is supposed to do, i.e., make more costly the ex post stranding of a technology which is revealed to be inferior.

It is interesting to note that Scharfstein and Stein (1990) recommend a similar mechanism to alleviate the "yes man" effect in the internal decision-making process in a firm. In their model of herd behavior, career concerns require that managers echo the predecessor's opinion in a committee meeting if voting is sequential. They suggest simultaneous casting of votes to avoid this problem.

\section{Concluding Remarks}

I have presented a simple model of technology adoption in which the interaction of network externalities and informational spillover generates risk aversion and herding in the choice of technology. We conclude with a discussion of limitations and possible extensions of our model.

First of all, the assumption on information generation and its transmission is too simple to be realistic. The true values of technologies may be revealed over time only through their repeated use. If users are diverse in their preferences, the values of the

\footnotetext{
${ }^{18}$ Standardization is also easy in the early stage of the technological cycle before potential users take any particular positions on the technologies. See Choi (1993) for more details and a formal analysis of the tradeoff identified. He provides a model of participants with homogeneous preferences in which standard-setting committees weigh the benefit of early standardization against the loss-of-sampling effect .
} 
technologies can be comprised of a common and an idiosyncratic (matching) component for each user. Then, we expect that the inference process for potential users who observe the experiences of previous users may be more complicated. As in the signal-jamming literature |see Fudenberg and Tirole (1986) and Riordan (1985)], there may also be incentives for committed users to manipulate information to induce subsequent users to choose their technology .

We have focused exclusively on the demand side of technology evolution by assuming competitively supplied technologies. However, the adoption behavior in the presence of network externalities and informational spillover can influence the supply side of the technologies as well. If the suppliers of the technologies have control over the choice of research strategies which affect the distribution of the values of technologies, they may adopt too safe a strategy in view of the risk-averse adoption behavior. ${ }^{19}$ Alternatively, suppliers of untested technologies may lease the capital goods that embody the technologies in an attempt to make the adoption decision (at least partially) reversible. However, this strategy will be effective in lessening the risk facing the early adopters only to the extent that the adoption of a new technology does not entail any investment in human capital and complementary goods.

Finally, it would be worthwhile to explore the implications of our model when the valuation of compatibility is asymmetric across users. Since the bias against the testing a new technology is driven by fears of being "orphaned," in our model the existence of users who are more or less independent of network externalities could mitigate the

${ }^{19}$ The standard result in the literature is that the market outcome is typically riskier than the socially optimal one [see Bhattacharya and Mookherjee (1986), Dasgupta and Maskin (1987), and Klette and de Meza (1986)]. Choi (forthcoming) provides another network externality-related reason for the possible reversal of the standard result. In his model with the waiting option, the choice of a less risky strategy by the sponsor of an emerging technology can induce consumers to wait until the technology is fully developed. Waiting can benefit the developer in two ways. First, she can have a larger market size in case it turns out that she has a better technology than her competitors. Second, there will not be the disadvantage of an installed base of competing technologies. 
underproduction of information. In the endogenous timing framework, we would expect these independent users to adopt earlier than those who are more concerned with network externalities since they are less affected by the potential stranding effect. Therefore, independent users could have a potentially positive effect on other users since they could prevent inefficient risk-averse behavior in the early adoption stages. Scharfstein and Stein (1990), in contrast, suggest that those with the stronger reputational concerns choose first in their model of herd behavior where the inefficiency originates from managers' reputational concerns. The reason is that in their paper there is no distortion for the initial decision-maker since the information externality only flows forward. This difference once again highlights the importance of backward externalities that information diffusion can invoke with payoff interdependence among agents. 


\section{References}

Aghion, P., P. Bolton, C. Harris, and B. Jullien, "Optimal Learning by Experimentation," Review of Economic Studies, 58 (1991), 621-654.

Arrow, K. J., "The Economic Implications of Learning-by-Doing." Review of Economic Studies, 29 (1962), 155-173.

Banerjee, A.V., "A Simple Model of Herd Behavior," Quarterly Journal of Economics, CVII (1992), 797-817.

Bhattacharya, S. and D. Mookherjee, "Portfolio Choice in Research and Development," Rand Journal of Economics, 17 (1986), pp.594-605.

Caplin, A. and J. Leahy, "Miracle on Sixth Avenue: Information Externalities and Search," H.I.E.R. Discussion Paper Series No.1665, 1993a.

, "Mass Layoffs and Unemployment," H.I.E.R.

Discussion Paper Series No. 1666, $1993 \mathrm{~b}$.

, "Business as Usual, Market Crashes, and Wisdom after the Fact," American Economic Review, forthcoming.

Chamley. C. and D. Gale, "Information Revelation and Strategic Delay in a Model of Investment," Econometrica, forthcoming.

Choi, J., "IrTeversible Choice of Uncertain Technologies with Network Externalities, Rand Journal of Economics, forthcoming.

. "Standardization and Experimentation: Ex Ante vs. Ex Post

Standardization," mimeo, CentER for Economic Research, 1993.

Dasgupta, P. and E. Maskin, "The Simple Economics of Research Portfolios," Economic Journal, 97 (1987), 587-595.

Farrell, J. and G. Saloner, "Standardization, Compatibility, and Innovation." Rand Journal of Economics, 16 (1985), 70-83.

, "Installed Base and Compatibility: Innovation, Product Preannouncements, and Predation, American Economic Review; 76 (1986), 940-955.

, "Competition, Compatibility, and Standards: The Economics of Horses. Penguins and Lemmings," in Product Compatibility as a Competitive Strategy, ed. H. L. Gabel., 1987, Amsterdam: North Holland.

. "Coordination through Committees and Markets."Rand Journal of Economics, 19 (1988), 235-252.

Fudenberg. D. and J. Tirole, "A "Signal-Jamming Theory of Predation." Rand Journal of Economics, 17 (1986), 366-376. 
Gabel, H. L., Competitive Strategies for Product Standards, 1991, Mc-Graw Hill.

Grossman, S., R.E. Kihlstrom and L.J. Mirman, "A Bayesian Approach to the Production of Information and Learning by Doing," Review of Economic Studies, 44 (1977), 533-547.

Katz, M. and C. Shapiro, "Technology Adoption in the Presence of Network Externalities," Journal of Political Economy, 94 (1986), 822-841.

Kislev, Y., and V. Shchori-Bachrach, "The Process of Innovation Cycle," American Journal of Agricultural Economics, LV (1973), 28-37.

Klette, T. and D. de Meza, " Is the Market Biased against Risky R\&D?" Rand Journal of Economics, 17 (1986), 133-139.

Lifchus, I. M., "Standards and Innovation: the Hidden Synergy", in Telecommunications and Equity, ed. J. Miller, 1986, 179-184, Amsterdam: North Holland.

Riordan, M.. "Imperfect Information and Dynamic Conjectural Variations," Rand Journal of Economics, 16 (1985) 41-50.

Rob, R., "Learning and Capacity Expansion under Demand Uncertainty," Review of Economic Studies, 58 (1991), 655-675.

Rosenberg, N., Inside the Black Box: Technology and Economics," Cambridge University Press, 1982.

Rothschild, M. and J. Stiglitz, "Increasing Risk: I. A Definition," Journal of Economic Theory, 2 (1970), 225-243.

Scharfstein, D., and J. Stein, "Herd Behavior and Investment," American Economic Review, LXXX (1990), 465-479.

Zeira, J., "Investment as a Process of Search," Journal of Political Economy, 95 (1986), 204-210. 


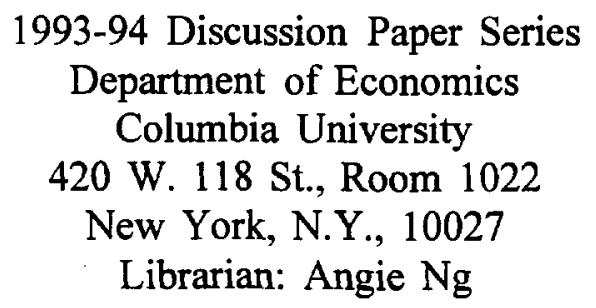

The following papers are published in the 1993-94 Columbia University Discussion Paper series which runs from November 1 to October 31. Domestic orders for discussion papers are available for purchase at $\$ 5.00$ (U.S.) each and $\$ 140.00$ (U.S.) for the series. Foreign orders cost $\$ 8.00$ (U.S.) for individual paper and $\$ 185.00$ for the series. To order discussion papers, please send your check or money order payable to Department of Economics, Columbia University to the above address. Please be sure to include the series number for the paper when you place an order.

671. Investment in U.S. Education and Training

Jacob Mincer ( Nov. 1993)

672. Freer Trade and the Wages of the Unskilled: Is Marx Striking Again?

Jagdish Bhagwati and Vivek Dehejia

673. Employer Size and Labor Turnover

Todd Idson

674. Less Crime May Be Worse

Brendan O'Flaherty

675. Team Production Effects on Earnings

Todd Idson

676. Language, Employment, and Earnings in the United States:

Spanish-English Differentials from 1970 to 1990

David Bloom and Gilles Grenier

677. The Impact of Performance Incentives on Providing Job Training to the Poor: The Job Training to the Poor: The Job Training Partnership

Act (JTPA)

Michael Cragg

678. The Demands to Reduce Domestic Diversity among Trading Nations Jagdish Bhagwati

679. Mass Layoffs and Unemployment Andrew Caplin and John Leahy 
680. The Economics of Adjustment

Andrew Caplin and John Leahy

681. Miracle on Sixth Avenue: Information Externalities and Search Andrew Caplin and John Leahy

682. Arbitrage, Gains from Trade and Scoial Diversity: A Unified Perspective on Resource Allocation Graciela Chichilnisky

683. Who should abate carbon emissions?

Graciela Chichilnisky, Geoffrey Heal

684. Believing in Multiple Equilibria Graciela Chichilnisky

685. Limited Arbitrage, Gains from Trade and Arrow's Theorem Graciela Chichilnisky

686. International Emission Permits: Equity and Efficiency Graciela Chichilnisky, Gecffrey Heal and David Starrett

687. Do Vehicle Emissions Testing Program Improve Air Quality? Matthew Kahn

688. Sources of Real Exchange Rate Fluctuations: How Important Are Nominal Shocks? Richard Clarida and Jordi Gali

689. Modeling Soviet Agriculture for Assessing Command Economy Policies Padma Desai and Balbir Sihag

690. The Changing Labor Market Position of Canadian Immigrants David Bloom, Gilles Grenier and Morley Gunderson

691. Herd Behavior, the " Penguin Effect ", and the Suppression of Informational Diffusion: An Analysis of Informational Externalities and Payoff Interdependency Jay Pil Choi 